\title{
Erythema Annulare Centrifugum and Relapsing Polychondritis Associated with Acute Myeloid Leukemia: A Case Report
}

\author{
Erythema Annulare Centrifugum ve Tekrarlayan Polikondritle \\ Birliktelik Gösteren Akut Miyeloid Lösemi: Bir Olgu Sunumu
}

\author{
Selami K. Toprak ${ }^{1}$, Sema Karakuş ${ }^{1}$, Aysun Halaçoğlu ${ }^{1}$, Efe Hasdemir ${ }^{1}$, Halit Üner ${ }^{2}$ \\ ${ }^{1}$ Baskent University, School of Medicine, Department of Hematology, Ankara, Turkey \\ ${ }^{2}$ Baskent University, School of Medicine, Department of Pathology, Ankara, Turkey
}

\section{To the Editor,}

Erythema annulare centrifugum (EAC) is a slow progressing and recurring skin disease with unknown etiology, characterized by ring shaped erythematous skin rashes. EAC was first described by Darier in 1916, and classified in 1978 by Ackerman into a superficial and a deep type [1]. EAC has been associated with many different entities, including infections, food allergy and drug reactions, polycythemia vera, cryoglobulinaemia, myelodysplastic syndrome, hypereosinophilic syndrome, hyperthyroidism, autoimmune thyroidities, hepatic diseases, pregnancy and malignant neoplasms [2]. Relapsing polychondritis (RP) is a disease which progresses with inflammatory attacks on articular and non-articular cartilaginous tissue and is most commonly encountered as auricular chondritis. In auricular chondritis, that the earlobe is not affected and existence of one or two sided fulminant rubescence, sensitivity and oedema on external ear cartilaginous tissue are salient [3]. We report a case, who was diagnosed with acute myeloblastic leukemia (AML) two months after EAC was detected. Bilateral auricular chondritis developed in the third day of her chemotherapy and EAC lesions relapsed during the course of chemotherapy.

A 53 year old female patient with complaints of a sore throat was assessed in otorhinolaryngology polyclinic.
While diagnosed with tonsillitis and her antibiotherapy arranged, she was redirected to us upon detection of anemia, moderate thrombocytopenia and leukocytosis (hemoglobin $11.2 \mathrm{~g} / \mathrm{dL}$, hematocrit $33 \%$, mean corpuscular volume $91 \mathrm{fL}$, white blood cell $19.2 \times 10^{9} / \mathrm{L}$, neutrophile 0.9 x $10^{9} / \mathrm{L}$, thrombocyte $120 \times 10^{9} / \mathrm{L}$ ). In her physical examination no symptom was found except cryptic tonsillitis. She did not have a known systemic disease nor she did regularly use a medication. Her liver, kidney and thyroid functions tests were normal. In her bone marrow biopsy, conducted upon monitoring of blastic cell with a rate of $90 \%$ during her peripheral smear, diffuse blastic cell infiltration was found. In bone marrow aspiration, usually large, potential undifferentiated blasts with heterogenous structure and agranular cytoplasm, but not involving Auer rods, were detected. After immunohistochemical examination the patient was diagnosed with AML M0, and classic $7+3$ induction chemotherapy regimen involving cytosine arabinoside and idarubicin combination protocol was initiated. On the third day of treatment palpable rash on her abdominal skin (Figure 1), and swelling, rubescence and sensitivity on both ears that do not prominently effect the earlobe (Figure 2a -2b) occurred. A biopsy from the lesions on the abdominal skin was performed. Lesions on the ear auricle of the patient were assessed as 


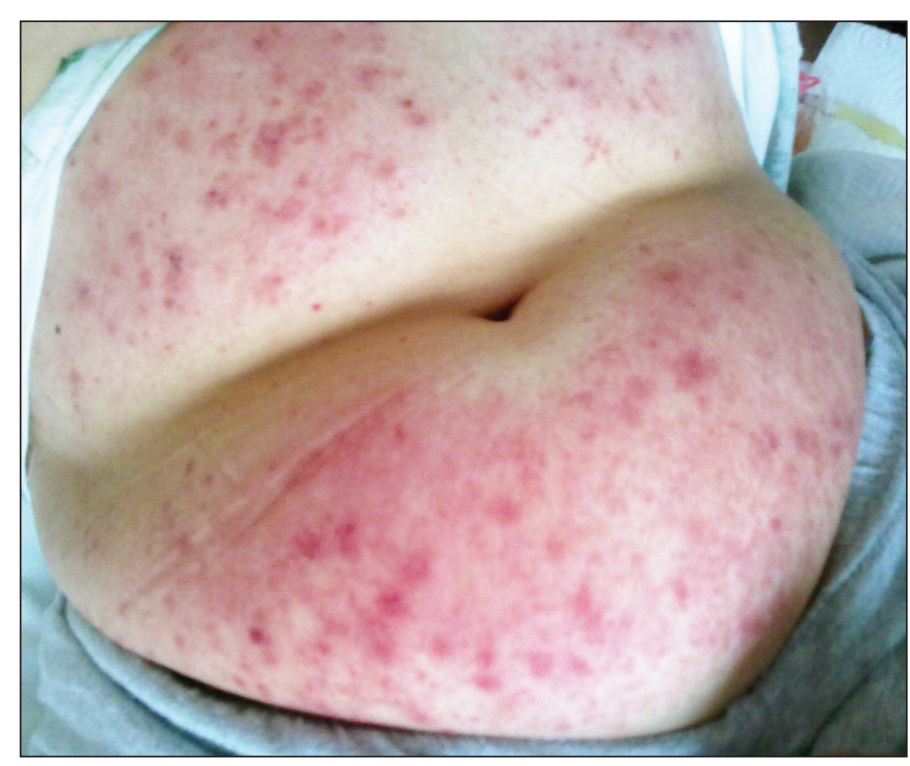

Figure 1: EAC lesions on the abdomen that occurred during the course of chemotherapy. chondritis by dermatology department and corticosteroid (CS) treatment (methylprednisolone $40 \mathrm{mg} /$ day) was initiated. However, while chondritis regressed on the third day of CS treatment, no change was detected on lesions of unknown cause (probably disease related or adverse effect of drugs?), which developed on the abdominal skin. Results of biopsy taken from abdominal skin a couple of days later came as EAC (Figure 3). When auricular chondritis started to evidently regress under treatment, CS treatment was decreasingly ceased within seven days. During the time when remission was achieved by means of bone marrow assessment carried out on the 21 st day of the course of chemotherapy, it was found that the rashes on the abdominal skin, which previously did not respond to CS treatment, decreasingly disappeared. It was also found that lesions similar to those on abdominal skin also developed on her both legs two months before the patient was diagnosed with AML and the results of biopsy that had
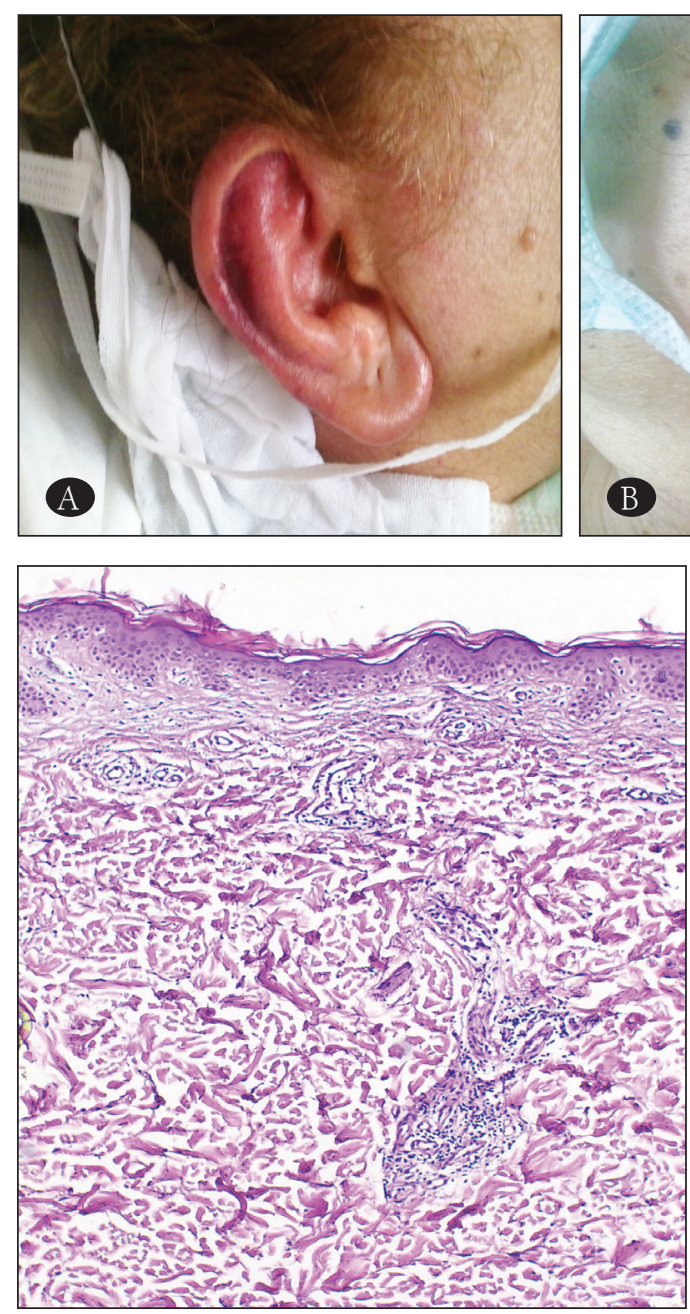

Figure 3: Benign, self-limited lymphocytic infiltration around the veins in superficial and deep dermis.
Figure 2A and 2B: Bilateral auricle and external auditory canal erythema, induration, and edema.

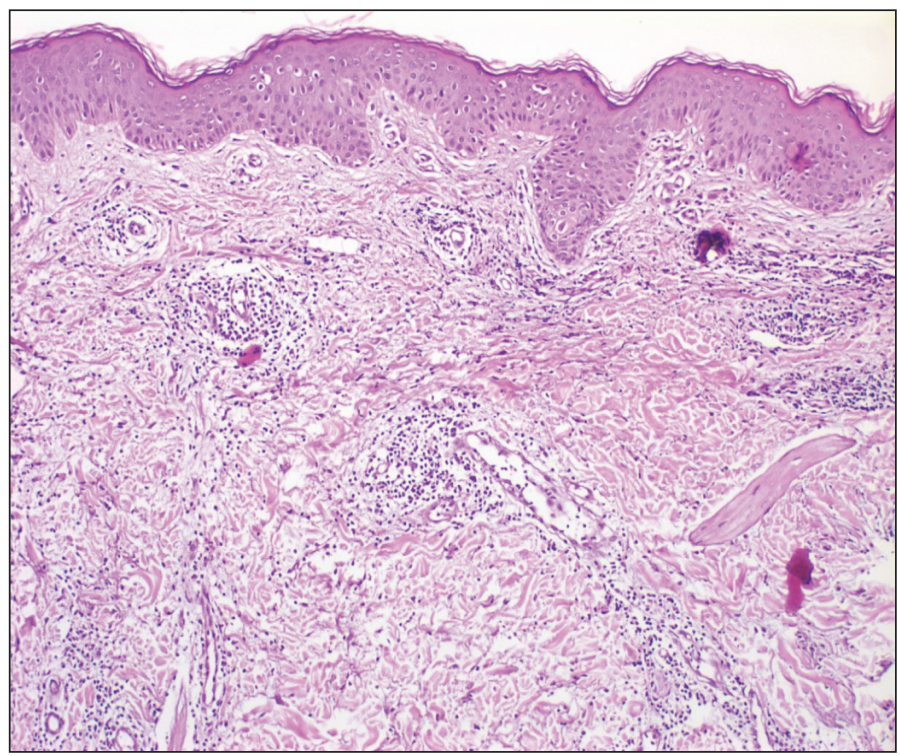

Figure 4: Prominent lymphoid infiltration around the veins in the dermis and among collagen fibers. 
been performed were likewise available as EAC (Figure 4). The patient is currently in remission and is being monitored in the course of allogeneic hematopoietic stem cell transplantation to follow consolidation chemotherapy.

Malignant diseases associated with EAC include myelomatosis, Hodgkin's lymphoma, acute leukemia, prostatic adenocarcinoma, nasopharyngeal carcinoma and histiocytosis [4].

In a study where 66 cases with EAC were assessed it was found that $13 \%$ of these were related to malignancies and at the same time there was no enough response to topical and systemic CS treatment [5]. In literature there is a case with anaplastic large cell lymphoma where a close relationship between the activity of disease and EAC was found [4]. Likewise, while our case did not respond to CS treatment, with the disease regression, skin lesions prominently regressed similarly to limited literature information.

Diagnostic algorithms for RP were established in 1976 by McAdam et al. These are; a) bilateral auricular chondritis, b) nonerosive, seronegative inflammatory polyarthritis, c) nasal chondritis, d) ocular inflammation, e) chondritis of respiratory system, and f) audiovestibular damage. Therefore, at least three criterion validities or an histopathological diagnosis and a criterion validity, or , along with response to CS and/or dapsone, existence of chondritis on two or more anatomic regions are sufficient for establishing a diagnosis of recurring polychondritis [6]. Our patient had bilateral auricular chondritis and she responded to CS. Our patient did not show any symptoms such as cough or dyspnoea that would suggest respiratory system cartilaginous tissue involvement. Additionally, no symptom regarding cartilaginous tissue involvement was found in her thorax computerized tomography taken when she had fever. No ocular, nasal or inner ear involvement was detected in our patient. While in RP skin involvement is most commonly encountered as vasculitis, erythema nodosum or superficial thrombophlebitis, when the literature was examined it was found that there is only one case where recurring polychondritis and EAC were detected $[7,8]$. Therefore, since our patient had the risk of cartilage involvement on a different anatomic region, we planned her follow-up also in this respect.

EAC is currently considered to be a distinctive hypersensitivity reaction that may be triggered by many different antigens and disappears after 1-2 weeks. Lesions tend to appear on the trunk, proximal parts of the extremities and, more frequently, in the superficial type appear especially on the buttocks [2].This is a chronic and recurrent disease despite treatment. EAC is thought to be highly associated with internal disease as well as with superficial fungal infection. However, it was difficult to prove a causal association. The recognition and exact diagnosis of EAC is important.

Another issue was the relationship between AML and EAC in our case. It is not clear whether these represent two separate diseases (AML and EAC) or a continuous range. The skin eruption may be the presenting sign of an underlying disease. In most cases, the etiology of EAC is unknown. However, numerous skin lesions may occur during AML. They more frequently have a nonleukemic infiltrate, mainly represented by eruption secondary to chemotherapeutic agents and antibiotics. Cutaneous AML due to skin infiltration by malignant blasts (leukemia cutis) is less frequent. For the present case; clinicopathological and molecular evidence was not suggestive of cutaneous AML. Many pathological associations have been reported in EAC, mainly in isolated clinical case reports [9]. Similarly, in a publication of Weyers et al., pathological associations were less frequent and patients with neoplasm-associated EAC were on average 20 years older than those with isolated EAC [1]. This might suggest a fortuitous association between cancer and EAC or at least an overestimation of this association mainly due to the fact that pathological associations with EAC tend to be more reported [9]. Nevertheless, in our patient, despite her young age, the low frequency of EAC in the general population and the concomitant discovery of EAC and AML argue against a fortuitous association.

The presented case is important because she was diagnosed with AML M0 only two months after EAC diagnosis and that auricular chondritis was detected alongside EAC on the third day of remission-induction chemotherapy. The probability of an underlying hematologic malignancy, especially in cases where EAC is detected, should always be kept in mind.

Written informed consent was obtained from the patient.

\section{Conflict of interest statement}

None of the authors of this paper has a conflict of interest, including specific financial interests, relationships, and/or affiliations relevant to the subject matter or materials included. 


\section{References}

1. Weyers W, Diaz-Cascajo C, Weyers I. Erythema annulare centrifugum: results of a clinicopathologic study of 73 patients. Am J Dermatopathol 2003; 25 (6): 451-462

2. Carlesimo M, Fidanza L, Mari E, Pranteda G, Cacchi C, Veggia B, Cox MC, Camplone G. Erythema annulare centrifugum associated with mantle b-cell non-Hodgkin's lymphoma. Acta Derm Venereol 2009; 89 (3): 319-320

3. Rapini RP, Warner NB. Relapsing polychondritis. Clin Dermatol 2006; 24 (6): 482-485

4. Ural AU, Ozcan A, Avcu F, Kaptan K, Taştan B, Beyan C, Yalçin A. Erythema annulare centrifugum as the presenting sign of CD 30 positive anaplastic large cell lymphoma-association with disease activity. Haematologia (Budap) 2001; 31 (1): 81-84

5. Kim KJ, Chang SE, Choi JH, Sung KJ, Moon KC, Koh JK. Clinicopathologic analysis of 66 cases of erythema annulare centrifugum. J Dermatol 2002; 29 (2): 61-67
6. McAdam LP, O'Hanlan MA, Bluestone R, Pearson CM. Relapsing polychondritis: Prospective study of 23 patients and a review of the literature. Medicine (Baltimore) 1976; 55 (3): 193-215

7. Ananthakrishna R, Goel R, Padhan P, Mathew J, Danda D. Relapsing polychondritis--case series from South India. Clin Rheumatol 2009; 28 Suppl 1: 7-10

8. Ingen-Housz S, Venutolo E, Pinquier L, Cavelier-Balloy B, Dubertret L, Flageul B. Erythema annulare centrifugum and relapsing polychondritis Ann Dermatol Venereol 2000; 127 (8-9): 735-739

9. Stokkermans-Dubois J, Beylot-Barry M, Vergier B, Bouabdallah K, Doutre MS. Erythema annulare centrifugum revealing chronic lymphocytic leukaemia. Br J Dermatol 2007; 157 (5): 1045-1047 